Centre for Decision Research and Experimental Economics

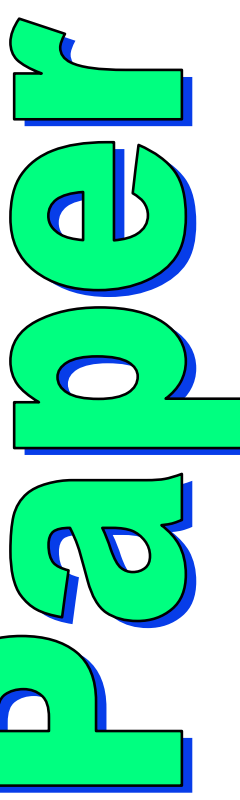

Discussion Paper Series

ISSN 1749-3293

CeDEx Discussion Paper No. 2008-03

Proportional Payoffs in Majority Games

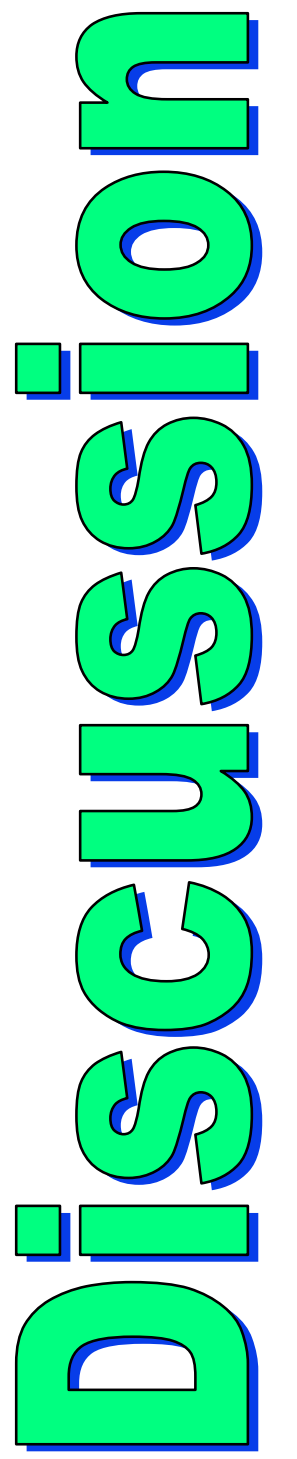

Maria Montero

March 2008 
The focus for the Centre is research into individual and strategic decision-making using a combination of theoretical and experimental methods. On the theory side, members of the Centre investigate individual choice under uncertainty, cooperative and non-cooperative game theory, as well as theories of psychology, bounded rationality and evolutionary game theory. Members of the Centre have applied experimental methods in the fields of Public Economics, Individual Choice under Risk and Uncertainty, Strategic Interaction, and the performance of auctions, markets and other economic institutions. Much of the Centre's research involves collaborative projects with researchers from other departments in the UK and overseas.

Please visit http://www.nottingham.ac.uk/economics/cedex/ for more information about the Centre or contact

Karina Whitehead

Centre for Decision Research and Experimental Economics

School of Economics

University of Nottingham

University Park

Nottingham

NG7 2RD

Tel: +44 (0) 1159515620

Fax: +44 (0) 1159514159

karina.whitehead@nottingham.ac.uk

The full list of CeDEx Discussion Papers is available at

http://www.nottingham.ac.uk/economics/cedex/papers/index.html 


\title{
Proportional Payoffs in Majority Games
}

\author{
Maria Montero*
}

March 14, 2008

\begin{abstract}
This paper extends the Baron-Ferejohn model of legislative bargaining to general weighted majority games with two modifications: first, payoff division can only be agreed upon after the coalition has formed (two-stage bargaining); second, negotiations in the coalition can break down, in which case a new coalition may be formed (reversible coalitions). Under the most natural bargaining protocol, both expected payoffs and actual payoff division are proportional to the voting weights provided that the set of winning coalitions of minimum weight is weakly balanced and that the breakdown probability tends to 0 . Homogeneity of the voting weights is neither necessary nor sufficient for proportional payoffs. Intermediate values of the breakdown probability produce predictions consistent with the empirical evidence on portfolio allocation in Europe: a moderate propoper advantage and a linear relationship between weights and ex post payoffs for all coalition members other than the proposer.
\end{abstract}

Keywords: coalition formation, legislative bargaining, weighted majority games, proportional payoffs, reversible coalitions.

J.E.L. classification: C71, C72, C78.

${ }^{*}$ School of Economics, University of Nottingham, University Park, NG7 2RD Nottingham (United Kingdom); e-mail: maria.montero@nottingham.ac.uk. Correspondence with Peter Sudhölter is gratefully acknowledged. A previous version appeared as Econ WPA 0308001. 


\section{Introduction}

The Baron-Ferejohn (1989) model is the most frequently used formal model of legislative bargaining. In this model, there are $n$ identical legislators, and decisions are taken by simple majority. An agent is selected at random (each agent with probability $1 / n$ ) to propose a division of the budget. If a majority votes in favor of the proposal, the proposal is implemented and the game ends (closed rule); otherwise, a new proposer is selected at random, and the process continues until an agreement is reached.

Even though coalitions are not explicitly formed in this model, we can think of the set of players who vote yes as the coalition that forms. Given this interpretation of the model, players are able to agree simultaneously on coalition formation and payoff division. ${ }^{1}$ However, there are situations in which players form tentative coalitions and then begin negotiations over payoff division. This paper separates the coalition formation and the payoff division stages, and follows Diermeier et al. (2003) in defining a coalition (a proto-coalition in their language) as a set of parties that agree to talk to each other about forming a government together. Negotiations over payoff division may break down, resulting in the dissolution of the coalition and possibly in the formation of a new one. ${ }^{2}$ The model also allows for general voting rules.

Proposers are randomly chosen both at the coalition formation and at the payoff division stage. The question then arises of what the proposer probabilities should be. It seems natural to assume that each of the players is chosen to be the proposer with a probability proportional to its number of votes. Under this assumption, I show that expected payoffs are proportional both ex ante (for all players) and ex post (for the members of the coalition) provided that the set of winning coalitions with the lowest total number of votes is weakly balanced and that the breakdown probability is arbitrarily

\footnotetext{
${ }^{1}$ This property is shared by other noncooperative models of coalition formation (see Selten (1981) and Chatterjee et al. (1993)).

${ }^{2}$ Diermeier et al. (2003) do not allow negotiations within a proto-coalition to break down. Another difference is that in their model the "cake" available to the proto-coalition depends on the state of the world, which evolves stochastically.
} 
close to 0 .

If the breakdown probability is allowed to vary, we obtain a family of models with different degrees of proposer advantage. If breakdown happens for sure after a proposal is rejected, the model is very close to the original Baron-Ferejohn model. The proportionality result still holds ex ante for all players and ex post for all coalition members other than the proposer. The proposer has an advantage that increases in the breakdown probability.

Perhaps surprisingly, homogeneity of the voting weights is neither necessary nor sufficient for proportionality of payoffs. The game being constant sum is sufficient provided that one chooses the "right" weights to represent the game.

The remainder of the paper is organized as follows. Section 2 introduces the bargaining procedure and some general properties of equilibria. Section 3 contains the proportionality result. Section 4 contains a discussion of the classes of games for which proportionality is obtained, and section 5 concludes.

\section{The game}

Let $N=\{1,2, \ldots, n\}$ be the set of players (parties) and $(N, v)$ a characteristic function game. The game $(N, v)$ is a weighted majority game iff there are $n+1$ nonnegative numbers $w_{1}, \ldots, w_{n}, q$ such that $v(S)=1$ if $\sum_{i \in S} w_{i} \geq q$ and 0 otherwise. The pair $[q ; w]$ where $w=\left(w_{1}, \ldots, w_{n}\right)$ is a representation of the game. We say that $S$ is winning iff $v(S)=1$, and that $S$ is minimal winning if $S$ is winning and no $T \varsubsetneqq S$ is winning. Given a weighted majority game, we will denote the set of winning coalitions as $W$ (or $W(q, w)$ if we want to emphasize that $W$ corresponds to $[q ; w]$ ), and the set of minimal winning coalitions as $W^{m}$. A player that belongs to all winning coalitions is called a veto player. A player that does not belong to any minimal winning coalition is called a dummy player.

We will interpret the characteristic function as referring to a budget of size 1 that has to be divided by majority rule. The characteristic function $v$ indicates which coalitions of parties have a majority (and thus can divide 
the budget between themselves). We will assume that all players in $N$ care only about their own material payoff and are risk neutral; thus if $x \in \mathbb{R}^{n}$ is a division of the budget $u_{i}(x)=x_{i}$.

Given the underlying weighted majority game, bargaining proceeds as follows:

- Nature selects a proposer according to a probability distribution $\theta$ $\left(\theta_{i} \geq 0\right.$ for all $i$ and $\left.\sum_{i \in N} \theta_{i}=1\right)$.

- The selected proposer $i$ proposes a coalition $S$ such that $S \ni i$.

- Players in $S$ accept or reject the proposal sequentially. If one of them rejects, Nature selects a new proposer according to the probability distribution $\theta$.

- If all players in $S$ accept, coalition $S$ is formed. If $S$ is a losing coalition, the game ends and all players get $0 .{ }^{3}$ If $S$ is a winning coalition, players in $S$ bargain over the division of the budget.

- The "internal" game, played only by players in $S$, is a bargaining game with random proposers (Nature follows a probability distribution $\theta^{S}$ with $\theta_{i}^{S} \geq 0$ for all $i \in S$ and $\sum_{i \in S} \theta_{i}^{S}=1$ ) and breakdown probability. A proposal $x^{S}$ is a division of the budget between the players in $S$ $\left(\sum_{i \in S} x_{i}^{S}=1\right)$. Every time a responder rejects a proposal, coalition $S$ is dissolved with probability $1-p(0<p<1)$.

- If coalition $S$ is dissolved, Nature selects a proposer again according to the probability distribution $\theta$.

We denote the noncooperative bargaining game described above as $G\left(N, v, \theta,\left(\theta^{S}\right)_{S \in W}, p\right)$, or simply $G$. We will be interested in stationary subgame perfect equilibria (SSPE).

Since coalition formation occurs before payoff division, we can think of the extensive game described above as a two-stage game. Of course, both the

\footnotetext{
${ }^{3}$ We may assume that $S$ leaves the game but $N \backslash S$ can continue negotiating without changing the results.
} 
coalition formation "stage" and the payoff division "stage" are complicated objects and the play may bring the players back from the second to the first stage.

We will refer to the probability distribution $\theta$ as the protocol, and to $\theta^{S}$ as the internal protocol. Given a SSPE $\sigma^{*}$, we will denote by $y$ the expected equilibrium payoff vector computed before Nature starts the game, and by $y^{S}$ the expected equilibrium payoff vector computed after $S$ has formed and before Nature starts the internal game. Let $z^{S}$ be the vector of continuation values (i.e., expected payoffs after a proposal has been rejected) in the internal game. Notice that because of stationarity, $y, y^{S}$ and $z^{S}$ depend on $\sigma^{*}$ but not on history. We start by computing the equilibrium of the internal game.

\subsection{The equilibrium of the internal game}

Suppose we have a SSPE of the game with associated expected equilibrium payoff vector $y$. We now show that the internal game has a unique stationary subgame perfect equilibrium payoff vector $y^{S}$.

If a player rejects a proposal in the internal game, with probability $p$ Nature starts the internal game again (so that player $i$ expects to get $y_{i}^{S}$ ) and with probability $1-p$ coalition $S$ breaks apart and Nature starts the coalition formation game again (so that player $i$ expects to get $y_{i}$ ). We have the following equation for the continuation value of player $i$ :

$$
z_{i}^{S}=p y_{i}^{S}+(1-p) y_{i}
$$

As for $y_{i}^{S}$, it is given by the probability that $i$ is selected to be a proposer in the internal game times his expected payoff as a proposer plus the probability that he is selected to be the responder (which is $1-\theta_{i}^{S}$ because 
bargaining is unanimous in the internal game) times his continuation value. ${ }^{4}$

$$
y_{i}^{S}=\theta_{i}^{S}\left[1-\sum_{j \in S \backslash\{i\}} z_{j}^{S}\right]+\left(1-\theta_{i}^{S}\right) z_{i}^{S}
$$

From this system of equations (and taking into account that $\sum_{j \in S} y_{j}^{S}=$ 1) we see that

$$
y_{i}^{S}=\theta_{i}^{S}\left(1-\sum_{j \in S} y_{j}\right)+y_{i}
$$

This is a well-known result in bargaining games with breakdown probability: player $i$ 's expected payoff equals the breakdown payoff (in this case, $y_{i}$ ) plus a share of the surplus proportional to the probability of being proposer (cf. Binmore (1987) and Binmore et al. (1986)).

Equation (1) resembles an allocation rule in that each player receives a share of the available surplus and this share is determined by the internal protocol. However, there is an important difference: the payoff a player gets from being in a coalition is not fully determined by the rules of the internal game because the breakdown outcome is endogenous, as in Rubinstein and Wolinsky (1985).

\subsection{The equilibrium of the game}

\subsubsection{No-delay result}

Under relatively weak conditions, agreement is reached immediately (cf. Okada, 1996).

Lemma 1 If $\theta_{i}<1$ for all $i, \theta_{i}^{S}>0$ for all winning coalitions $S \ni i$ and there are no veto players, then in any SSPE of $G$ all proposals are accepted, and a winning coalition forms immediately.

\footnotetext{
${ }^{4}$ The payoff for player $i$ as a proposer is $1-\sum_{j \in S \backslash\{i\}} z_{j}^{S}$ regardless of whether agreement is immediate. There are two possible cases:

1) If $\sum_{i \in S} z_{i}^{S}<1$ the proposer strictly prefers making acceptable proposals; since in any SSPE the proposer must offer exactly $z_{j}$ to each responder $j$ the results holds.

2) If $\sum_{i \in S} z_{i}^{S}=1$ acceptable and unacceptable proposals give the same payoffs to the players. Notice that this second case is only possible if $\sum_{i \in S} y_{i}^{S}=1$ and $\sum_{i \in S} y_{i}=1$.
} 
Proof. The first thing to notice is that $y_{i} \geq 0$ because no player can be forced into a coalition. A proposal to form a coalition $S$ with $\sum_{k \in S} y_{k}<1$ is always accepted because the payoff from accepting the proposal, $y_{j}+\theta_{j}^{S}(1-$ $\left.\sum_{k \in S} y_{k}\right)$, is strictly greater than the payoff from rejecting it, $y_{j}$, for all $j \in N .{ }^{5}$ Consider the situation of $i$ as proposer. There is always a coalition $i$ can propose with $1>\sum_{k \in S} y_{k}$. This is because $N \backslash\{k\}$ is winning for all $k$, thus player $i$ can propose any $N \backslash\{k\}$ where $k$ is such that $y_{k}>0$. The only case in which this would not be possible is if $y_{i}=1$, but clearly this cannot happen in equilibrium because all other players would propose coalitions without $i$ (and such coalitions would be accepted), resulting in $i$ getting 0 with positive probability, contradicting $y_{i}=1$. Since $\theta_{i}^{S}>0$ for all $S$, proposing $S$ is strictly better than proposing a losing coalition.

On the equilibrium path, $i$ proposes a winning coalition $S$ with $\sum_{k \in S} y_{k}<$ 1 and bargaining between players in $S$ results in immediate agreement.

Corollary 2 If moreover $\theta_{i}>0$ for all $i$, then in any SSPE of $G$ we have $y_{i}>0$ for all $i$.

Proof. Clearly, a player will never accept a negative payoff in any situation. By rejecting all proposals and proposing only losing coalitions, a player can secure a payoff of 0 . Furthermore, as a proposer, any player gets a positive surplus because there is a coalition $S \ni i$ with $1>\sum_{k \in S} y_{k}$.

Corollary 3 The proposer will propose a coalition $S$ that solves the following problem

$$
\max _{W \ni S \ni i} \theta_{i}^{S}\left(1-\sum_{k \in S} y_{k}\right)
$$

Because the solution of this problem is sure to have $\sum_{k \in S} y_{k}<1$, the proposer does not need to worry about acceptance.

Corollary 4 Let $\lambda_{i}^{S}$ be the probability that player $i$ proposes coalition $S$. Under the conditions of lemma 1, the following must hold in any SSPE of

\footnotetext{
${ }^{5}$ Notice that equilibria in which several responders reject just because $S$ is going to be rejected anyhow are ruled out by the fact that the players in $S$ respond sequentially.
} 
$G$ :

$$
\begin{aligned}
y_{i} & =\theta_{i} \sum_{S \ni i} \lambda_{i}^{S}\left[\theta_{i}^{S}\left(1-\sum_{k \in S} y_{k}\right)+y_{i}\right]+\sum_{j \in N \backslash\{i\}} \theta_{j} \sum_{S \supset\{i, j\}} \lambda_{j}^{S}\left[\theta_{i}^{S}\left(1-\sum_{k \in S} y_{k}\right)+y_{i}\right] \\
\sum_{S \ni i} \lambda_{i}^{S} & =1 \\
\lambda_{i}^{S} & >0 \text { implies } S \in \arg \max _{T: T \ni i} \theta_{i}^{T}\left(1-\sum_{j \in T} y_{j}\right)
\end{aligned}
$$

\subsubsection{How coalitions larger than minimal winning may form}

If we make no further assumptions on $\theta$ and $\left(\theta_{S}\right)_{S \in W}$, proposed coalitions are not necessarily minimal winning.

Suppose $\theta_{i}^{T}>\theta_{i}^{S}$ for some $T \supset S$. Then player $i$ is facing a tradeoff: $i$ receives a higher share of the surplus in $T$, but the surplus of $T$ is smaller. Example 5 shows that the first effect may predominate. This is not completely obvious because $\left(y_{j}\right)_{j \in N}$ is endogeneously determined.

Example 5 Consider the apex game with seven players $[6 ; 5,1,1,1,1,1,1]$. Suppose $\theta_{1}=\frac{7}{8}, \theta_{i}=\frac{1}{48}, \theta_{i}^{N \backslash\{1\}}=\frac{1}{6}$ and $\theta_{1}^{S}=\frac{s-1}{s}$, where $s$ is the number of players in $S .{ }^{6}$ There is an equilibrium in which the large player forms a coalition with two small players.

Let the large player propose to two small players at random, thus each small player has a probability $\frac{2}{6}$ of receiving a proposal if the large player is selected to be proposer. Let the small players propose coalition $N \backslash\{1\}$. Given these strategies, the equilibrium payoffs can be found from the following equations:

$$
\begin{aligned}
& y_{1}=\frac{7}{8}\left[y_{1}+\frac{2}{3}\left(1-y_{1}-2 y_{n}\right)\right] \\
& y_{n}=\frac{7}{8} \frac{2}{6}\left[y_{n}+\frac{1}{6}\left(1-y_{1}-2 y_{n}\right)\right]+\frac{1}{8} \frac{1}{6}
\end{aligned}
$$

The solution is $y_{1}=\frac{28}{37}, y_{n}=\frac{3}{74}$. Player 1 is behaving optimally because $\frac{s-1}{s}\left[1-y_{1}-(s-1) y_{n}\right]$ is maximized for $s=3$. The small players are also

${ }^{6}$ The value $\frac{s-1}{s}$ is also the coalition structure Shapley value (Aumann and Drèze, 1974) of player 1 for any $S$ with $\{1\} \varsubsetneqq S \neq N$. 
behaving optimally by proposing coalition $N \backslash\{1\}$. In $N \backslash\{1\}$, they expect a payoff of $\frac{1}{6}$. If instead they proposed $\{1, i\}$, they would get a payoff of $\frac{3}{74}+\frac{1}{2}\left(1-\frac{28}{37}-\frac{3}{74}\right)=\frac{21}{148}<\frac{1}{6}$.

\subsubsection{Games with veto players}

If there are veto players, the two-stage feature of the model does not make any difference and the set of equilibria is the same as in the original BaronFerejohn game without discounting. ${ }^{7}$ Assuming that all players have a positive probability of being proposers, all non-veto players get 0 and any division of the total payoff between the veto players is an equilibrium regardless of the protocol. Agreement may be delayed but must eventually occur.

\section{The proportionality result}

We now turn to the question of proportionality of payoffs. Because each game has many equivalent representations ${ }^{8}$, for this section we use $[q ; w]$ and not $(N, v)$ as the primitive of the analysis. We will not make any assumptions about $q$ and $w$ for the time being, except $q>\frac{\sum_{i \in N} w_{i}}{2}$ (so that it is not possible for two disjoint sets of players to achieve a majority). There is no presumption that $[q ; w]$ is the canonical representation of the game. Without loss of generality, we will normalize the weights so that $\sum_{i \in N} w_{i}=1$.

The question we ask is the following. Suppose $\theta$ and $\left(\theta^{S}\right)_{S \in W}$ are proportional to the voting weights. Is there an equilibrium with proportional payoffs? More specifically, is there an equilibrium in which expected payoffs coincide with $w$ and expected payoffs conditional on $S$ forming are proportional to $w$ ?

\footnotetext{
${ }^{7}$ Games with veto players are studied by Winter (1996); see also appendix A in Drouvelis, Montero and Sefton (2007).

${ }^{8}$ Two representations, $[q, w]$ and $\left[q^{\prime}, w^{\prime}\right]$, are equivalent if they have the same set of winning coalitions, i.e., $W(q, w)=W\left(q^{\prime}, w^{\prime}\right)$.
} 


\subsection{Discarding dummy players}

We have started from a very general $[q ; w]$. In particular, we haven't excluded the possibility of dummy players with a small but positive voting weight. The following lemma shows that, if we are interested in proportional payoffs under a proportional protocol, we can ignore dummy players without any loss of generality: if payoffs are proportional to $w, w$ cannot give a positive weight to dummy players.

Lemma 6 If $\theta_{i}=w_{i}$, and $\theta_{i}^{S}=\frac{w_{i}}{\sum_{j \in S} w_{j}}$ for every $S \in W$ and $i \in S$, $y_{i}=w_{i}$ for a dummy player $i$ implies $w_{i}=0$.

Proof. Suppose $w_{i}=y_{i}>0$ for a dummy player $i$. No player $j \neq i$ will include $i$ in the proposed coalition (dropping $i$ increases the surplus available to the coalition as well as the share $j$ receives of the surplus). Let $T$ be one of the coalitions that are optimal for $i$ as a proposer. Then

$$
y_{i}=\theta_{i}\left[y_{i}+\theta_{i}^{T}\left(1-\sum_{j \in T} y_{j}\right)\right]
$$

Given the assumption of propotional protocol and proportional expected payoffs, we can write the equation as

$$
w_{i}=w_{i}\left[w_{i}+\frac{w_{i}}{\sum_{j \in T} w_{j}}\left(1-\sum_{j \in T} w_{j}\right)\right]=w_{i} \frac{w_{i}}{\sum_{j \in T} w_{j}} .
$$

Since there must be some nondummy players in $T$, we have $w_{i}<\sum_{j \in S^{*}} w_{j}$ and the equation cannot hold.

Remark 7 Since proportionality of payoffs under a proportional protocol requires dummy players to get 0 , we will henceforth assume that there are no dummy players, or equivalently we will assume $w_{i}=0$ for all dummies and redefine the set of players $N$ as the set of those that belong to at least one minimal winning coalition. This will simplify the statements below. 


\subsection{A sufficient condition for proportionality}

Let $\mathcal{C}$ be a collection of nonempty subsets of $N$. We say that the collection is (weakly) balanced ${ }^{9}$ iff there exist (nonnegative) positive numbers $\left(\lambda_{S}\right)_{S \in \mathcal{C}}$ such that, for each $i \in N, \sum_{S \ni i} \lambda_{S}=1$. The numbers $\left(\lambda_{S}\right)_{S \in \mathcal{C}}$ are called balancing weights.

Given any $(q, w)$, denote by $W^{*}(q, w)$ the collection of winning coalitions with minimum total weight, namely

$$
W^{*}(q, w):=\left\{S \in W: w_{S} \leq w_{T} \text { for all } T \in W\right\} .
$$

Clearly, $W^{*}(q, w) \subseteq W^{m}(q, w)$, that is, winning coalitions of minimum total weight must be minimal winning coalitions. If no confusion arises, we will denote $W^{*}(q, w)$ by $W^{*}$. We will denote $\min _{S \in W} w_{S}$ by $q^{*} \cdot{ }^{10}$

The main result of this paper is that, if the voting weights are such that the set of winning coalitions with minimum total weight is weakly balanced, and the recognition probabilities are proportional to the voting weights, then expected payoffs (ex ante and conditional on the coalition that forms) are also proportional to the voting weights. Actual payoff division is proportional as well in the limit when the breakdown probability tends to 0 .

Proposition 8 Let $(q, w)$ be a weighted majority game such that $W^{*}(q, w)$ is a weakly balanced collection. Let $\theta_{i}=w_{i}$, and $\theta_{i}^{S}=\frac{w_{i}}{\sum_{j \in S} w_{j}}$ for every $S \in$ $W$ and $i \in S$. Then there exists an SSPE of the game $G\left(q, w, \theta,\left(\theta^{S}\right)_{S \in W}, p\right)$ in which

(1) Only coalitions in $W^{*}(q, w)$ are proposed.

(2) Expected payoffs are $y_{i}=w_{i}$ for all $i$ in $N$.

(3) Expected payoffs conditional on $S$ forming are $y_{i}^{S}=\frac{w_{i}}{\sum_{j \in S} w_{j}}$ for all coalitions $S$ that form in equilibrium.

\footnotetext{
${ }^{9}$ This concept appears in Shapley (1967).

${ }^{10}$ Because we haven't assumed anything about $[q ; w], q^{*}>q$ is possible. For example, in the game $[11 ; 9,3,3,3,3], q^{*}=12$ but $q=11$. Any representation of the game with a claim to be the canonical representation would have $q=q^{*}$ (see Peleg 1968).
} 
(4) Ex post, each responder obtains $\left[\frac{p}{q^{*}}+1-p\right] w_{i}$, and the proposer obtains the residual. The proposer's payoff is decreasing in the continuation probability $p$ and varies between $1-\sum_{j \in S \backslash\{i\}} w_{j}$ and $\frac{w_{i}}{q^{*}}$.

Proof. Let $\left(\lambda_{S}\right)_{S \in W^{*}}$ be a set of balancing weights. Consider the following strategy combination:

- As a proposer, player $i$ proposes coalition $S$ with probability $\lambda_{S}$ if $S \in$ $W^{*}$ and $i \in S$, and with probability 0 otherwise. Since $\sum_{S \ni i} \lambda_{S}=1$, player $i$ 's strategy as a proposer is completely determined.

- As a responder, player $i$ accepts all proposals to form winning coalitions and rejects all proposals to form losing coalitions.

- Given that a coalition $S$ forms, the equilibrium of the internal game as described in section 2.1 is played.

In order for the above strategies to be an SSPE of the game, two conditions must be satisfied:

- Players are behaving optimally given the expected payoffs $\left(y_{i}\right)_{i \in N}$.

- Expected payoffs given the proposed coalitions are indeed $\left(y_{i}\right)_{i \in N}$.

To show that players are behaving optimally, suppose $y_{i}=w_{i}$ for all $i \in N$. By construction, players' strategies are an equilibrium of the internal game for any $S$. Since expected payoffs in the internal game are at least $y_{i}$ (see equation (1)), it is optimal to accept all proposals to form a winning coalition. Since all losing coalitions end up with a payoff of 0 , it is also optimal to reject all proposals to form a losing coalition. It remains to check that only optimal coalitions are proposed.

From corollary 3 , the proposer proposes a coalition that maximizes $\theta_{i}^{S}\left(1-\sum_{k \in S} y_{k}\right)$ over the set of winning coalitions to which it belongs. Thus, other things being equal, the proposer prefers to propose coalitions with a high $\theta_{i}^{S}$ and a low $\sum_{k \in S} y_{k}$. If expected payoffs are proportional to the weights, the coalitions that minimize $\sum_{k \in S} y_{k}$ are the same as those that 
maximize $\theta_{i}^{S}$, namely the coalitions in the set $W^{*}$. Weak balancedness of $W^{*}$ does not play a role here except to guarantee that each $i \in N$ belongs to at least one of those coalitions.

Recall that $q^{*}$ denotes the total weight of any coalition $S \in W^{*}$. Expected payoffs conditional on a coalition $S \in W^{*}$ forming are

$$
y_{i}^{S}=w_{i}+\frac{w_{i}}{q^{*}}\left[1-q^{*}\right]=\frac{w_{i}}{q^{*}} .
$$

This result is independent of the value of $p$. Actual payoff division is such that each responder receives its continuation value and the proposer pockets the residual. Continuation values are

$$
z_{i}^{S}=p \frac{w_{i}}{q^{*}}+(1-p) w_{i}=\left[\frac{p}{q^{*}}+1-p\right] w_{i}
$$

Thus, actual payoffs are proportional to $w_{i}$ for all responders, but the proposer obtains a disproportionate share. In the limit when the continuation probability tends to 1 , the proposer advantage vanishes and actual payoff division is proportional.

We have shown that players are behaving optimally given that $y_{i}=w_{i}$ for all $i \in N$ and that this behavior induces proportional values for $y_{i}^{S}$. It remains to show that ex ante expected payoffs induced by the strategies are indeed $\left(w_{i}\right)_{i \in N}$.

Because only coalitions in $W^{*}$ form given the strategies, $y_{i}^{S}=\frac{w_{i}}{q^{*}}$ for all coalitions $S \ni i$ that have a positive probability of forming. Thus, $y_{i}=\lambda_{i} \frac{w_{i}}{q^{*}}$, where $\lambda_{i}$ is the probability that player $i$ belongs to the coalition that forms. In order for the strategy combination described above to have $y=w$, we need $\lambda_{i}=q^{*}$ for all $i \in N$.

$$
\lambda_{i}=\sum_{S \in W^{*}, S \ni i} \sum_{j \in S} w_{j} \lambda_{S}=\sum_{S \in W^{*}, S \ni i} \lambda_{S} \sum_{j \in S} w_{j}=\sum_{S \in W^{*}, S \ni i} \lambda_{S} q^{*}=q^{*} .
$$

When is $W^{*}(q, w)$ weakly balanced? In order to answer this question, 
consider the following linear programming problem ${ }^{11}$

$$
\begin{aligned}
\text { s.t. } \sum_{i \in S} x_{i}+e & \geq 1 \text { for all } S \in W \\
\sum_{i \in N} x_{i} & =1 \\
x_{i} & \geq 0 \text { for all } i
\end{aligned}
$$

The value $1-\sum_{i \in S} x_{i}$ is the surplus (usually called the excess) available if coalition $S$ forms. The program solves for vectors of nonnegative payoffs that minimize the maximum $1-\sum_{i \in S} x_{i}$. The nucleolus (Schmeidler, 1969) is a solution of this program by definition.

The following lemma is a weak version of a much stronger result proven by Kohlberg (1971).

Lemma 9 The set $W^{*}(q, w)$ is weakly balanced iff the weight vector $w$ is a solution of the above program.

Proof. The dual of the linear program above (after noticing that we can replace $\sum_{i \in N} x_{i}=1$ by $-\sum_{i \in N} x_{i} \geq-1$ without changing the solution) is

$$
\begin{aligned}
& \max \sum_{S \in W} \lambda_{S}-\mu \\
& \sum_{W \ni S \ni i} \lambda_{S}-\mu+\rho_{i} \leq 0 \\
& \sum_{S \in W} \lambda_{S} \leq 1 \\
& \lambda_{S} \geq 0 \text { for all } S \in W, \rho_{i} \geq 0 \text { for all } i \text { and } \mu \geq 0
\end{aligned}
$$

where $\lambda_{S}$ is the dual variable associated to the constraint $x(S)+e \geq 1$, $\mu$ is the dual variable associated to $-x(N) \geq-1$ and $\rho_{i}$ is the dual variable associated to $x_{i} \geq 0$.

\footnotetext{
${ }^{11}$ We could also write the constraints as $\sum_{i \in S} x_{i}+e \geq v(S)$ for all $S$. This would make no difference because the constraints corresponding to losing coalitions would not be binding.
} 
Suppose $w$ is a solution to the primal. Complementary slackness implies that, for any coalition with $\lambda_{S}>0$, we have $x(S)+e=1$, that is, $S$ is a coalition of maximum excess (or of minimal total weight). Because $w_{i}>0$ for all players (we are ignoring dummies!), $\rho_{i}=0$ and $\sum_{S \ni i} \lambda_{S}=\mu$ for all $i \in N$. If the core is empty, the value of $e$ that solves the problem is positive and complementary slackness implies $\sum_{S \in W} \lambda_{S}=1$. It is then clear that $\mu>0$ and we can define $\lambda_{S}^{\prime}=\frac{\lambda_{S}}{\mu}$. Then $\sum_{W \ni S \ni i} \lambda_{S}^{\prime}=1$ for all $i$ and the result follows. If the core is nonempty (i.e., if there are veto players), $N$ is a coalition of maximum excess and setting $\lambda_{N}=1$ shows that $W^{*}$ is weakly balanced.

Conversely, if $W^{*}(q, w)$ is weakly balanced and $N \in W^{*}, w$ is in the core and is clearly a solution to the program. If $N \notin W^{*}$ we can show that $w$ solves the program by constructing feasible solutions to the primal and to the dual satisfying complementary slackness. For the primal, simply compute the maximum excess $e^{*}$ associated to $w$. For the dual, $W^{*}(q ; w)$ only contains winning coalitions. Given a set $\left(\lambda_{S}^{\prime}\right)_{S \in W^{*}}$ of balancing weights, define $\lambda_{S}:=\frac{\lambda_{S}^{\prime}}{\sum_{S \in W} \lambda_{S}^{\prime}}$ if $S \in W^{*}, \lambda_{S}=0$ if $S \notin \mathbf{W}^{*}, \rho_{i}=0$ for all $i$ and $\mu:=\sum_{S \ni i} \lambda_{S}^{\prime}$. One can check that $\left(w, e^{*}\right)$ is feasible for the primal, $\left(\left(\lambda_{S}\right)_{S \in W}, \mu\right)$ is feasible for the dual and complementary slackness holds. By the complementary slackness theorem (cf. Theorem 5.3 in Vanderbei (2001)) we have a solution to the program.

\section{Discussion}

\subsection{On the relation between $W^{*}(q, w)$ being weakly balanced and other properties of games}

A given game may have many equivalent representations. For example, $[2 ; 1,1,1]$ and $[5 ; 4,3,2]$ are equivalent representations of the same game. Not all representations will have the property that $W^{*}$ is a weakly balanced collection. In the previous example, $W^{*}(2 ; 1,1,1)=\{\{1,2\},\{1,3\},\{2,3\}\}$, and this collection is weakly balanced (each minimal winning coalition can be given a weight of $\left.\frac{1}{2}\right)$. On the other hand, $W^{*}(5 ; 4,3,2)=\{\{2,3\}\}$, which 
is clearly not weakly balanced.

We may ask ourselves whether, given any majority game, we can find an equivalent representation such that $W^{*}$ is weakly balanced and if so, whether this representation is unique. The answer to both questions is negative in general. If the game is constant-sum, there is always a representation such that the associated $W^{*}$ is weakly balanced. If the game is moreover homogeneous, this representation is unique. Homogeneity on its own is unrelated to whether $W^{*}$ is weakly balanced.

\section{Games with veto players}

Games with veto players do not satisfy the balancedness condition unless all players are veto players, in which case proposition 8 holds trivially.

\section{Constant-sum homogeneous games}

A game is constant-sum if $v(S)+v(N \backslash S)=1$ for all $S \subset N$ (no ties are possible). It is homogeneous if we can find a representation $\left[q^{h} ; w^{h}\right]$ such that $v(S)=1$ iff $\sum_{i \in S} w_{i}^{h} \geq q^{h}$ and $\sum_{i \in S} w_{i}^{h}=q^{h}$ for all $S \in W^{m}$.

For this class of games, the nucleolus is a representation and the only solution to the linear programming problem (see Peleg, 1968). Thus, there is a unique system of weights such that expected payoffs with a proportional protocol are themselves proportional.

\section{Constant-sum but not homogeneous games}

The nucleolus is still a representation (Peleg, 1968) but it may not be the only solution to the linear programming problem. Any solution to the linear programming problem is also a representation.

The following example illustrates how proportional payoffs can be achieved despite the lack of homogeneity of the game.

Example 10 Consider the game $[5 ; 2,2,2,1,1,1]$. Normalizing the weights so that they add up to 1 we get $w=\left(\frac{2}{9}, \frac{2}{9}, \frac{2}{9}, \frac{1}{9}, \frac{1}{9}, \frac{1}{9}\right)$ and $q=\frac{5}{9}$. The set $W^{*}(q, w)$ is weakly balanced.

Given $w=\left(\frac{2}{9}, \frac{2}{9}, \frac{2}{9}, \frac{1}{9}, \frac{1}{9}, \frac{1}{9}\right)$, there are two types of coalition of maximum excess: [221] and [2111]. This is a balanced collection, since we can assign a weight of $\frac{2}{15}$ to each of the nine coalition of type [221] and $\frac{1}{5}$ to each of the three coalitions of type [2111]. In the noncooperative game, players would 
propose coalitions to which they belong with a probability equal to the corresponding balancing weight. Coalition $\{1,2,3\}$ is also minimal winning but does not form because it has a lower surplus to divide $\left(\frac{3}{9}\right.$ as opposed to $\left.\frac{4}{9}\right)$ and its members receive a lower share of this surplus ( $\frac{1}{3}$ as opposed to $\left.\frac{2}{5}\right)$.

The only difference with the homogeneous case is that, if there are several solutions of the linear programming problem, there are several payoff vectors $w$ with the property that a proportional protocol to $w$ yields $w$ as expected payoff vector.

Isbell (1959) has an example of two representations of the same game: $[99 ; 38,31,31,28,23,12,11,8,6,5,3,1]$ and $[99 ; 37,31,31,28,23,12,11,8,7,5,3,1]$. Both of them minimize the maximum excess, thus proposition 8 applies to both. The nucleolus is the midpoint.

\section{Homogeneous but not constant-sum games}

If $(q ; w)$ is a homogeneous representation, the set $W^{*}$ is precisely the set of minimal winning coalitions. Theorem 8 applies to $w$ whenever $W^{m}$ is weakly balanced. ${ }^{12}$

There are homogeneous games for which the set of minimal winning coalitions is not weakly balanced. For example, in game $[5 ; 3,2,2,1]$, the set of minimal winning coalitions is $\{\{1,2\},\{1,3\},\{2,3,4\}\}$, which is not balanced. Since player 4 belongs to only one of these coalitions, we would need to set $\lambda_{\{2,3,4\}}=1$, which would imply $\lambda_{\{1,2\}}=\lambda_{\{1,3\}}=0$ (otherwise the weights would add up to more than 1 for players 2 and 3). But then $\sum_{S \ni 1} \lambda_{S}=0$.

The example above is of a game with "steps". A step is a player that cannot be replaced by smaller players in a minimal winning coalition. "Steps rule their followers" (Ostmann, 1987, lemma 4.8) in the sense that they must be members of any minimal winning coalition in which a smaller player appears.

One may ask whether the set of minimal winning coalitions is weakly

\footnotetext{
${ }^{12}$ Note that if the set of minimal winning coalitions is not weakly balanced, the set $W^{*}(q, w)$ cannot be weakly balanced for any $w$ (homogeneous or otherwise). This is because $W^{*}(q, w) \subset W^{m}(q, w)$.
} 
balanced for homogeneous games without steps. The answer is again not in general (see Peleg and Rosenmüller, 1992).

Example 11 Consider the game $[18 ; 4,4,4,4,2,2,2,1,1,1]$. This is a game without steps, but the set of minimal winning coalitions is not weakly balanced.

One may check this directly by setting a system of equations for the balancing weights $\left(\lambda_{S}\right)_{S \in W^{*}}$ and showing that there is no solution with $\lambda_{S} \geq 0$ for all $S \in W^{*}$. Another way is to notice that if $W^{*}$ was weakly balanced, then $w$ would be a solution to the linear programming problem in lemma 9. However, there is another vector with a lower maximum excess: $\left(\frac{1}{4}, \frac{1}{4}, \frac{1}{4}, \frac{1}{4}, 0,0,0,0,0,0\right)$. The maximum excess associated to this imputation is $\frac{1}{4}$; for the homogeneous representation it is $1-\frac{18}{25}=\frac{7}{25}>\frac{1}{4}$.

There are also many homogeneous games for which $W^{m}$ is weakly balanced. Then there is a set of weights $w$ for which theorem 8 holds. This set is not necessarily unique, as the following example shows.

Example 12 Consider the game $[6 ; 2,2,2,1,1,1]$. The set of minimal winning coalitions is weakly balanced, thus the weight vector $w=\left(\frac{2}{9}, \frac{2}{9}, \frac{2}{9}, \frac{1}{9}, \frac{1}{9}, \frac{1}{9}\right)$ satisfies the conditions of theorem 8. The nonhomogeneous weight vector $\left(\frac{1}{4}, \frac{1}{4}, \frac{1}{4}, \frac{1}{12}, \frac{1}{12}, \frac{1}{12}\right)$ (which happens to be the nucleolus) $)^{13}$ also does.

\section{Games that are neither homogeneous nor constant-sum}

The possibility of $W^{*}$ being weakly balanced is not excluded.

Example 13 Consider the game $[8 ; 4,3,2,2,1,1]$. The set $W^{*}(q, w)$ is weakly balanced.

The set $W^{*}(q, w)$ contains all winning coalitions with exactly 8 votes, namely two coalitions of type [431], one coalition of type [422], two coalitions of type [4211] and two coalitions of type [3221]. We can assign a weight of $\frac{3}{16}$ to each coalition of type [431], $\frac{1}{8}$ to the coalition of type [422], $\frac{1}{4}$ to each coalition of type [4211] and $\frac{5}{16}$ to each coalition of type [3221].

\footnotetext{
${ }^{13} \mathrm{~A}$ useful computer program that calculates the nucleolus has been developed by Derks and Kuipers (1997).
} 


\subsection{On the impossibility of fully proportional payoffs for some games}

There is a sense in which the present model yields full proportional payoffs whenever feasible. Abstracting from any particular model, suppose ex post payoffs for all coalitions are proportional to the voting weights. It seems natural to assume that only minimal winning coalitions with the lowest total voting weight form, since they are the coalitions that maximize their members' payoffs ${ }^{14}$. Let $q^{*}$ be the total weight of any coalition in $W^{*}$. Conditional on being in the final coalition, each player is receiving $\frac{w_{i}}{q^{*}}$. Ex ante expected payoffs equal $\lambda_{i} \frac{w_{i}}{q^{*}}$ for each player, where $\lambda_{i}$ is the probability that $i$ is included in the coalition that forms. Ex ante proportionality can only be achieved if all players are in the final coalition with the same probability. In other words, the set of coalitions that form, which is the set $W^{*}$, must be weakly balanced, otherwise proportionality ex ante and proportionality ex post are incompatible.

The impossibility we have just discussed is on the assumption that only coalitions in $W^{*}$, which are the most attractive ones if ex post payoffs are proportional, can form. Coming back to our particular model, we saw in section 2 that it is possible to design protocols such that coalitions larger than minimal winning can form. Perhaps proportional payoffs can be obtained with a protocol that induces larger coalitions. This is indeed the case, but the protocol has to be degenerate in the sense that at least one player is not allowed to propose coalitions. ${ }^{15}$

Example 14 Consider the game $[5 ; 3,2,2,1]$. Ex ante and ex post SSPE payoffs can only be simultaneously proportional if there is a player $i$ with $\theta_{i}=0$.

Proof. We know from the previous discussion that proportional payoffs cannot be achieved if only minimal winning coalitions form. There are three

\footnotetext{
${ }^{14}$ This is the logic behind Gamson's (1961) model of coalition formation.

${ }^{15}$ For the original Baron-Ferejohn model, Kalandrakis (2006) finds that any ex ante expected payoffs can be obtained for some protocol (which may assign a probability of 0 to some players).
} 
coalitions larger than minimal winning that could form in a hypothetical equilibrium: $\{1,2,4\},\{1,3,4\}$ and $\{1,2,3,4\}$. Suppose $\{1,2,3,4\}$ forms. Some player must have proposed it. If it is player 1 , it must be the case that 1 gets at most $\frac{3}{8}$ in $\{1,2\}$ and $\{1,3\}$. Then $\theta_{2}=\theta_{3}=0$ (otherwise these players would get $\frac{5}{8}$ or more as proposers). The same reasoning applies in general: if $i$ proposes a coalition larger than minimal winning, it must be that $i$ is badly treated by the internal protocol in the minimal winning coalitions to which $i$ belongs, and thus some other player $j$ must be generously treated. In order to preserve proportionality, $\theta_{j}=0$, or else $j$ would propose a coalition with $i$ or some other coalition that treats $j$ even better, and proportionality would break down.

There is always a trivial protocol that induces proportional payoffs: take a player $i$, and let $\theta_{i}=1$ and $\theta_{i}^{S}=0$ for all $S \neq N$. Then any payoff vector including $w$ is an equilibrium payoff vector.

\subsection{Making the formateur coincide with the first proposer}

The present model separates the role of formateur (the player selected to propose a coalition) and first proposer (the player selected to propose a division of the payoff, which may or may not be the formateur). ${ }^{16}$ The model predicts a proposer advantage (for $p<1$ ) rather than a formateur advantage. The results are very similar if the model is modified to make the first proposer coincide with the formateur. ${ }^{17}$ The model then predicts a formateur advantage that decreases in the continuation probability $p$.

Consider the following modified model: if player $i$ proposes $S$ and $S$ is accepted, player $i$ gets to make the first payoff division proposal. If this proposal is rejected, $\theta_{i}^{S}=\frac{w_{i}}{\sum_{j \in S} w_{j}}$ for all subsequent rounds until an agreement occurs or $S$ breaks down. The probability of being selected as formateur is still $\theta_{i}=w_{i}$ for all $i$.

Consider the internal game. After the first proposal is rejected, the game becomes identical to the one we have analyzed, thus if we denote

\footnotetext{
${ }^{16}$ For another model that separates the roles of formateur and proposer, see Seidmann et al. (2007).

${ }^{17}$ Diermeier et al. (2003) make this assumption.
} 
as $y_{i}^{S}$ the expected payoff conditional on $S$ forming and the first proposal having been rejected, the continuation values at the first round are still given by $z_{i}^{S}=p y_{i}^{S}+(1-p) y_{i}$ and the equation $y_{i}^{S}=y_{i}+\theta_{i}^{S}\left[1-\sum_{j \in S} y_{j}\right]$ still holds. In particular, if $y_{i}=w_{i}$, it still follows that $y_{i}^{S}=\frac{w_{i}}{q^{*}}$ for all $S \in W^{*}$, and each responder would receive $z_{i}^{S}=\left[\frac{p}{q^{*}}+1-p\right] w_{i}$ as in proposition 8 .

The same strategies as in proposition 8 lead to proportional payoffs. Coalitions in $W^{*}$ are still the optimal coalitions to propose given that $y_{i}=$ $w_{i}$. The only difference is that the equation for expected ex ante payoffs is no longer as simple as $y_{i}=\lambda_{i} \frac{w_{i}}{q^{*}}$ (the probability of being in the final coalition times expected payoff conditional on being in the final coalition) but it needs to separate the cases in which $i$ is a proposer (with probability $w_{i}$, and payoff $1-\sum_{j \in S \backslash\{i\}}\left[\frac{p}{q^{*}}+1-p\right] w_{j}$ for some $\left.S \in W^{*}\right)$ and cases in which $i$ is a responder (with probability $\sum_{W^{*} \ni S \ni i} \sum_{j \in S \backslash\{i\}} w_{j} \lambda_{S}$ and payoff $\left.\left[\frac{p}{q^{*}}+1-p\right] w_{i}\right)$. Given that $\sum_{j \in S \backslash\{i\}} w_{j}=q^{*}-w_{i}$ for all $S \in W^{*}$, i's payoff as a proposer can be written as $1-\left[\frac{p}{q^{*}}+1-p\right]\left(q^{*}-w_{i}\right)$, and the probability of $i$ being a responder can be written as $\left(q^{*}-w_{i}\right) \sum_{W^{*} \ni S \ni i} \lambda_{S}$. Since by construction $\sum_{W^{*} \ni S \ni i} \lambda_{S}=1$, the probability of being a responder is $q^{*}-w_{i}$. Then expected payoffs can be written as

$$
y_{i}=w_{i}\left[1-\left[\frac{p}{q^{*}}+1-p\right]\left(q^{*}-w_{j}\right)\right]+\left(q^{*}-w_{j}\right)\left[\frac{p}{q^{*}}+1-p\right] w_{i}=w_{i} .
$$

\section{Conclusion}

While proportionality (ex ante and ex post) is a very natural result it is not present in the literature to the best of my knowledge. Von Neumann and Morgenstern's (1944) main simple solution and other cooperative solution concepts like the aspiration core (Cross, 1967) and the demand bargaining set (Morelli and Montero, 2003) predict a proportional division of payoffs inside the coalition that actually forms, but have nothing to say about $e x$ ante expected payoffs. Ex ante solution concepts like the Shapley value do not predict proportional payoffs; the nucleolus assigns proportional payoffs to the grand coalition but not to minimal winning coalitions. Something 
similar can be said of noncooperative models: either proportionality holds only ex post (as in Frechette et al. 2005, Montero and Vidal-Puga 2006), or it holds only ex ante, while ex post the proposer gets a disproportionate payoff (as in Montero 2006). Here, the proposer's advantage vanishes in the limit when the probability of breakdown tends to 0 .

Empirical evidence on legislative bargaining finds a linear relationship between weights and payoffs for coalition members other than the formateur, and a significant formateur advantage, though this advantage is not quite as large as the original Baron-Ferejohn model would predict (see Ansolabehere et al., 2005). The current model with an intermediate breakdown probability and the formateur being the first proposer makes precisely these predictions. ${ }^{18}$

The condition of weak balancedness of the set $W^{*}$ is intuitive. It says that we can assign probabilities to the coalitions that form in such a way that each player is in the final coalition with the same probability. Ex post payoffs can be described as competitive, since none of the players is particularly popular or impopular given those payoffs. Interestingly, the weak balancedness condition is independent of other well-known properties such as homogeneity.

The present model yields fully proportional payoffs whenever feasible. If only minimal winning coalitions with the lowest total voting weight form (which seems natural if ex post payoffs are proportional to the weights), ex ante proportionality can only be achieved if all players have the same probability of being in the coalition that forms. In other words, the set of coalitions that form must be weakly balanced, otherwise proportionality ex ante and proportionality ex post are incompatible.

\footnotetext{
${ }^{18}$ One caveat is that it may not be possible to find an equivalent representation with the property that $W^{*}(q, w)$ is weakly balanced for some of the parliaments in the sample. Another is that $(q, w)$ may be quite different from the actual seat shares. Diermeier and Merlo (2004) find some empirical support for formateur selection proportional to seat shares; to the best of my knowledge formateur selection proportional to voting weights has not been tested. Ansolabehere et al. (2005) examine the question of which party succeeds in forming the government and find that minimum integer voting weights and seat shares separately predict who is the successful formateur.
} 


\section{References}

[1] Ansolabehere, S., Snyder, J.M., Strauss, A.B. and Ting, M. M. (2005). Voting weights and formateur advantages in the formation of coalition governments, American Journal of Political Science, vol. 49(3) (July), pp. 550-63.

[2] Aumann, R.J. and Drèze, J.H. (1974). Cooperative games with coalition structures. International Journal of Game Theory 3, 217-237.

[3] Baron, D. P., and Ferejohn, J. A. (1989) Bargaining in legislatures. American Political Science Review 83, 1181-1206.

[4] Binmore, K. (1987) Perfect equilibria in bargaining models. In: Binmore, K., Dasgupta, P. (eds.) The Economics of Bargaining. Blackwell, Oxford.

[5] Binmore, K., Rubinstein, A., and Wolinsky, A. (1986) The Nash bargaining solution in economic modelling. Rand Journal of Economics 17, $176-88$.

[6] Chatterjee, K., Dutta, B., Ray, D., and Sengupta, K. (1993) A noncooperative theory of coalitional bargaining. Review of Economic Studies 60, 463-77.

[7] Cross, J. (1967). Some theoretical characteristics of economic and political coalitions. Journal of Conflict Resolution 11, 184-195.

[8] Derks, J. and Kuipers, J. (1997). Implementing the simplex method for computing the prenucleolus of transferable utility games. Unpublished manuscript.

[9] Diermeier, D., Eraslan, H., and Merlo, A. (2003). A structural model of government formation. Econometrica 71, 27-70.

[10] Diermeier, D. and Merlo, A. (2004) An empirical investigation of coalitional bargaining procedures. Journal of Public Economics 88, 783-797. 
[11] Drouvelis, M., Montero, M. and Sefton, M. (2007). The paradox of new members: strategic foundations and experimental evidence. CeDEx Discussion Paper 2007-13.

[12] Fréchette, G., Kagel, J. and Morelli, M. (2005) Behavioral identification in coalitional bargaining: an experimental analysis of demand bargaining and Alternating Offers. Econometrica 73, 1893-1937.

[13] Gamson, W.A. (1961) A theory of coalition formation. American Sociological Review 26, 373-382.

[14] Isbell, J.R. (1959) On the enumeration of majority games. Mathematical Tables and Other Aids to Computation 13, 21-28.

[15] Kalandrakis, T. (2006) Proposal rights and political power. American Journal of Political Science 50, 441-448.

[16] Kohlberg, E. (1971) On the nucleolus of a characteristic function game. SIAM Journal of Applied Mathematics 20, 62-66.

[17] Montero, M. (2006) Noncooperative foundations of the nucleolus in majority games. Games and Economic Behavior 54, 380-397.

[18] Montero, M. and J. J. Vidal-Puga (2006) Demand bargaining and proportional payoffs in majority games. CeDEx Discussion Paper 2006-11.

[19] Morelli, M. and M. Montero (2003) The demand bargaining set: general characterization and application to weighted majority games. Games and Economic Behavior 42, 137-155.

[20] Okada, A. (1996) A noncooperative coalitional bargaining game with random proposers. Games and Economic Behavior 16, 97-108.

[21] Ostmann, A. (1987) On the minimal representation of homogeneous games. International Journal of Game Theory 16, 69-81.

[22] Peleg, B. (1968) On weights of constant-sum majority games. SIAM Journal of Applied Mathematics 16, 527-532. 
[23] Peleg, B. and J. Rosenmüller (1992) The least core, nucleolus, and kernel of homogeneous weighted majority games. Games and Economic Behavior 4, 588-605.

[24] Rubinstein, A. and A. Wolinsky. (1985) Equilibrium in a market with sequential bargaining. Econometrica 53, 1133-1150.

[25] Schmeidler, D. (1969) The nucleolus of a characteristic function game SIAM Journal on Applied Mathematics 17, 1163-1170.

[26] Seidmann, D.J., Winter, E. and Pavlov, E. (2007). The formateur's role in government formation. Economic Theory 31, 427-445.

[27] Selten, R. (1981) A noncooperative model of characteristic function bargaining. In: Böhm, V., Nachtkamp, H. H. Essays in Game Theory and Mathematical Economics. Bibliographisches Institut, Mannheim.

[28] Shapley, L.S. (1967). On balanced sets and cores. Naval Research Logistics Quarterly 14, 453-460.

[29] Vanderbei, R.J. (2001) Linear programming: foundations and extensions. London: Kluwer Academic.

[30] Winter, E. (1996) Voting and vetoing. American Political Science Review $90,813-823$.

[31] von Neumann, J. and O. Morgenstern (1944). Theory of Games and Economic Behavior. Princeton: Princeton University Press. 